\section{ORIGINAL RESEARCH}

C.H. Toh

K.-C. Wei

C.-N. Chang

P.-W. Hsu

H.-F. Wong

S.-H. $\mathrm{Ng}$

M. Castillo

C.-P. Lin

\title{
Differentiation of Pyogenic Brain Abscesses from Necrotic Glioblastomas with Use of Susceptibility-Weighted Imaging
}

BACKGROUND AND PURPOSE: A common imaging finding in brain abscess and necrotic glioblastoma is a T2 hypointense margin. The features of this hypointense rim on SWI have not been previously described, to our knowledge. We aimed to differentiate abscesses from glioblastomas by assessing the morphology of their lesion margin by using SWI.

\begin{abstract}
MATERIALS AND METHODS: T2WI and SWI were performed in 12 abscesses and 20 rim-enhancing glioblastomas. On T2WI and SWI, the prevalence and the border types (complete versus incomplete) of hypointense rims were qualitatively assessed. On SWI, the contour (smooth versus irregular) and the location of hypointense rims relative to the contrast-enhancing rims as well as the prevalence of the "dual rim sign," defined as 2 concentric rims at lesion margins with the outer one being hypointense and the inner one hyperintense relative to cavity contents, were also analyzed.
\end{abstract}

RESULTS: Prevalence and the border types of the hypointense rims on T2Wl were not different between abscesses and glioblastomas. On SWI, there were significantly more hypointense rims that were complete $(P<.001)$ and smooth $(P<.001)$, having the same location as the contrast-enhancing rims $(P<.001)$ for abscesses. A dual rim sign was present in 9 of 12 abscesses but absent in all glioblastomas $(P<.001)$.

CONCLUSIONS: SWI may be helpful in differentiating pyogenic abscesses from necrotic glioblastomas The dual rim sign is the most specific imaging feature distinguishing the 2 .

ABBREVIATIONS: GRAPPA = generalized autocalibrating partially parallel acquisition; mIP = minimum intensity projection; MPRAGE = magnetization-prepared rapid acquisition of gradient echo; SWI = susceptibility-weighted imaging

D WI that measures overall diffusivity in terms of directionally averaged ADC complements the role of conventional MR imaging in the differentiation of abscesses from necrotic glioblastomas, given that generally ADC is low in abscess cavities and high in tumor cysts. ${ }^{1}$ However, high ADC similar to that found in necrotic tumors has been reported in 5\%-21\% of untreated abscesses. ${ }^{2,3}$ On the other hand, necrotic glioblastomas may demonstrate low ADC mimicking abscesses. ${ }^{2,4,5}$ In short, differentiation of brain abscesses from necrotic glioblastomas with DWI can sometimes be difficult.

The presence of a hypointense rim at the lesion margin on FSE T2WI is a common feature of brain abscesses and necrotic glioblastomas. ${ }^{6,7}$ A previous study ${ }^{7}$ reported that in abscesses, the

\section{Received October 17, 2011; accepted after revision November 18.}

From the Departments of Medical Imaging and Intervention (C.H.T., H.-F.W., S.-H.N.) and Neurosurgery (K.-C.W., C.-N.C., P.-W.H.), Chang Gung Memorial Hospital, Linkou and Chang Gung University College of Medicine, Tao-Yuan, Taiwan; Department of Biomedical Imaging and Radiological Sciences (C.H.T., C.-P.L.) and Brain Connectivity Laboratory (C.H.T., C.-P.L.), Institute of Neuroscience, National Yang-Ming University, Taipei, Taiwan; and Department of Radiology (M.C.), University of North Carolina School of Medicine, Chapel Hill, North Carolina.

This work was supported by the Animal Molecular Imaging Center, Chang Gung Memorial Hospital at Linkou, Taiwan; and, in part, by grants from the National Science Council (NSC-98-2314-B-182A-051-MY3 to C.H. Toh).

Please address correspondence to Cheng Hong Toh, MD, Department of Medical Imaging and Intervention, Chang Gung Memorial Hospital, Linkou and Chang Gung University College of Medicine, No. 5, Fuxing St, Guishan Township, Taoyuan County 333, Taiwan; e-mail: eldomtoh@hotmail.com

Indicates open access to non-subscribers at www.ajnr.org

http://dx.doi.org/10.3174/ajnr.A2986 hypointense rims that tended to be "complete" bordered the lesions entirely, whereas gliomas more often featured hypointense rims that were "incomplete" and partially bordered the lesions.

SWI is a high-resolution 3D gradient-echo sequence that combines phase and magnitude information to detect magnetic susceptibility differences between adjacent tissues with a sensitivity greater than that in conventional gradient-echo sequences and T2WI. ${ }^{8}$ Its high sensitivity in the detection of paramagnetic and diamagnetic substances such as iron deposits, blood-degradation products, and calcifications provides clinically useful information in the evaluation of various disorders, including neurodegenerative disease, cerebral neoplasm, trauma, demyelination, vascular malformation, and intracranial hemorrhage. ${ }^{9}$

In pictorial essays illustrating the clinical utility of SWI ${ }^{10,11}$ brain abscesses were described as having a markedly hypointense rim. The contrast between the hypointense rims and the abscess cavities as well as the surrounding edema was higher compared with FSE T2WI. To our knowledge, to date, the prevalence and morphology of hypointense rims have never been studied in detail with SWI, and the role of SWI in brain abscess has not been evaluated. We aimed to differentiate abscesses from glioblastomas by assessing the morphology of their lesion margins by using SWI.

\section{Materials and Methods}

\section{Patients}

Approval for this study was obtained from the Institutional Board of Research Associates and signed informed consent was obtained from 

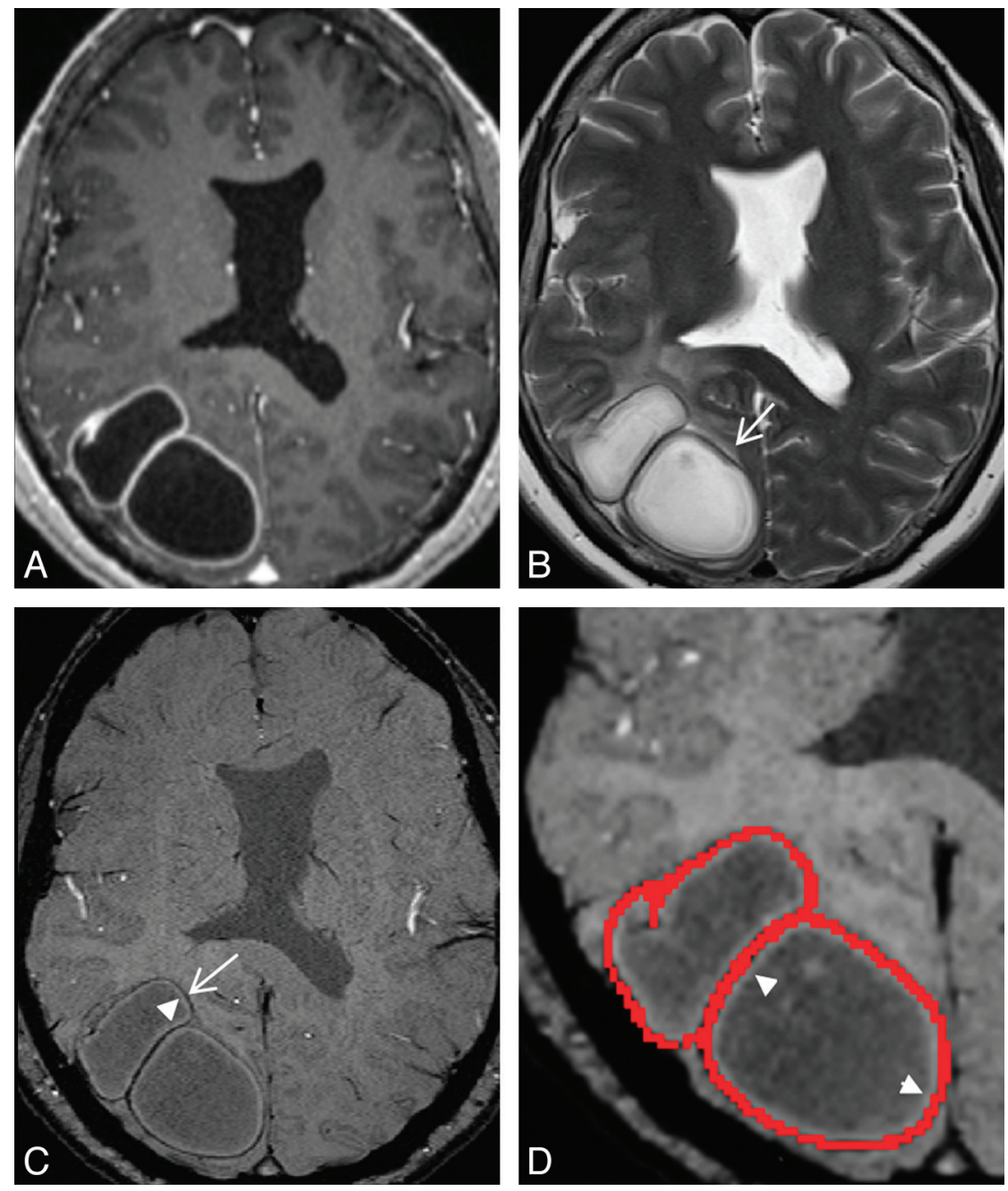

Fig 1. A 56-year-old woman with 2 right occipital pyogenic brain abscesses. A, Transverse contrast-enhanced MPRAGE shows 2 adjoining rim-enhancing masses in the right occipital lobe. $B$, On the transverse T2-weighted image, an incomplete hypointense rim (arrow) is present at the lesion margin. $C$, On transverse SWI, the abscesses are bordered by 2 concentric rims, with the outer one being hypointense (arrow), and the inner one, hyperintense (arrowhead) relative to cavity content, forming the dual rim sign. D, On the close-up view of the SWI with the contrast-enhancing rim (red region of interest) overlaid, the hyperintense rim (arrowhead) is inner to the contrast-enhancing rim.

all patients. This study was Health Insurance Portability and Accountability Act-compliant. Preoperative MR imaging studies were performed in 12 patients ( 7 men, 5 women; mean age, 56.1 years; age range, 34-74 years) with brain abscesses (mean size, $4.0 \pm 0.9 \mathrm{~cm}$ ) and 20 patients ( 16 men, 4 women; mean age, 59 years; age range, 27-91 years) with necrotic glioblastomas (mean size, $4.5 \pm 1.1 \mathrm{~cm}$ ). All lesions appeared as a rim-enhancing mass with perifocal edema on MR imaging. The diagnosis of brain abscess was surgically confirmed in all patients. All 12 abscesses were pyogenic. In 7 of 12 patients, causative agents were confirmed by cultures of aspirated pus. In 5 patients, no pathogen was isolated. However, Gram-positive cocci $(n=3)$ or Gram-negative bacilli $(n=2)$ were seen in smears of aspirated pus. Histologic diagnosis was obtained in all patients with necrotic glioblastomas. Three purely hemorrhagic, 5 solid, and 3 infiltrative glioblastomas were excluded.

\section{MR Imaging}

All MR imaging studies were performed by using a 3T unit (Magnetom Tim Trio; Siemens, Erlangen, Germany). All patients underwent MR imaging studies, including transverse T1WI, transverse FSE T2WI (TR/TE, 6200/98 ms; section thickness, $4 \mathrm{~mm}$; flip angle, $120^{\circ}$; echo-train length, 19; acquisition matrix, $314 \times 512$; FOV, $192 \times 220$ $\mathrm{mm}$ ), transverse SWI (TR/TE, 28/20 ms; section thickness, $1.2 \mathrm{~mm}$; flip angle, $15^{\circ}$; acquisition matrix, $298 \times 448$; FOV, $180 \times 230 \mathrm{~mm}$ ), and postcontrast 3D MPRAGE (TR/TE, 2000/2.63 ms; section thickness, $1 \mathrm{~mm}$; TI, $900 \mathrm{~ms}$; acquisition matrix, $224 \times 256$; FOV, $224 \times$ $256 \mathrm{~mm}$ ) with intravenous administration of $0.1 \mathrm{mmol}$ per kilogram body weight of gadopentetate dimeglumine (Magnevist; Schering, Berlin, Germany).

\section{Imaging Analysis}

The transverse FSE T2WI and the original SWI images without mIP were coregistered to postcontrast MPRAGE (Fig 1A) on the basis of $3 \mathrm{D}$ nonrigid transformation and mutual information before all imaging analysis with the use of SPM2 (Wellcome Department of Cognitive Neurology, London, United Kingdom). The adequacy of registration was visually assessed by a neuroradiologist, and manual adjustments were performed if necessary. Two experienced neuroradiologists blinded to the diagnosis evaluated all lesions independently. Interobserver differences were resolved by consensus.

Each lesion was reviewed for the presence of a hypointense rim, defined as a discernible border of hypointensity relative to the hyperintensity of the lesion center, first on FSE T2WI (Fig 1B) and then SWI (Fig 1C) 1 week apart. The border types of the hypointense rim were 


\begin{tabular}{|c|c|c|c|c|c|c|c|c|}
\hline \multicolumn{9}{|c|}{ Comparisons of the imaging features of the lesion margins on SWI between abscesses and glioblastomas } \\
\hline Features & Abscess & GB & $P$ Value & SEN & SPE & Accuracy & PPV & NPV \\
\hline \multicolumn{9}{|l|}{ Hypointense rim } \\
\hline Prevalence & & & 0.059 & & & & & \\
\hline Absent & 0 & 5 & & & & & & \\
\hline Present & 12 & 15 & & & & & & \\
\hline Border & & & $<.001$ & 91.7 & 86.7 & 88.9 & 84.6 & 92.9 \\
\hline Incomplete & 1 & 13 & & & & & & \\
\hline Complete ${ }^{\mathrm{a}}$ & 11 & 2 & & & & & & \\
\hline Contour & & & $<.001$ & 75.0 & 86.7 & 81.5 & 81.8 & 81.3 \\
\hline Smooth ${ }^{a}$ & 9 & 2 & & & & & & \\
\hline Irregular & 3 & 13 & & & & & & \\
\hline Location $^{\mathrm{b}}$ & & & $<.001$ & 91.7 & 86.7 & 88.9 & 84.6 & 92.9 \\
\hline Inner & 0 & 9 & & & & & & \\
\hline Same $^{\mathrm{a}}$ & 11 & 2 & & & & & & \\
\hline Inner + Same & 1 & 4 & & & & & & \\
\hline Dual rim sign & & & $<.001$ & 75.0 & 100 & 90.6 & 100 & 87.0 \\
\hline Absent & 3 & 20 & & & & & & \\
\hline Present $^{\mathrm{a}}$ & 9 & 0 & & & & & & \\
\hline
\end{tabular}

Note:-GB indicates glioblastoma; SEN, sensitivity; SPE, specificity; PPV, positive predictive value; NPV, negative predictive value.

a Imaging features selected for the calculation of SEN, SPE, PPV, and NPV.

${ }^{b}$ Relative to the contrast-enhancing rims

categorized as complete if the entire lesion was surrounded and incomplete when only a part of the lesion was surrounded. On SWI, we also analyzed the contour of the rims and defined these as smooth or irregular and assessed their locations relative to the contrast-enhancing rims. A threshold pixel value was manually chosen to create a scatter region of interest so as to segment the contrast-enhancing rim. The adequacy of segmentation was visually assessed by a neuroradiologist. The segmented contrast-enhancing rim was then overlaid onto the SWI to determine the locations of the hypointense rim (Fig $1 D)$. Finally, each lesion was assessed for the presence of the "dual rim sign" (Fig 1C). This sign was defined as 2 concentric rims surrounding the central cavities at lesion margins, with the outer one being hypointense and the inner one, hyperintense relative to the cavity contents.

\section{Statistical Analysis}

The prevalence and border types of hypointense rims on T2WI and SWI, the contours and locations of the hypointense rim, and the prevalence of the dual rim sign on SWI were examined by using a $\chi^{2}$ test. Sensitivity, specificity, accuracy, and positive and negative predictive values in differentiating abscesses from necrotic glioblastomas were calculated for each imaging feature with statistically significant differences. A commercially available statistical software package (Statistical Package for the Social Sciences, Version 16; SPSS, Chicago, Illinois) was used for analysis, and $P$ values $<.05$ were considered to indicate statistically significant differences.

\section{Results}

On T2WI, hypointense rims were present in 7 of $12(58.3 \%)$ abscesses, with $4(57.1 \%)$ being complete. Of the necrotic glioblastomas, 10 of 20 (50\%) had hypointense rims on T2WI, of which $8(80 \%)$ were incomplete. There were no statistically significant differences between abscesses and necrotic glioblastomas in the prevalence $(P=.647)$ as well as the border types $(P=.115)$ of their T2-weighted hypointense rims.

The results of comparisons of lesion margins on SWI between abscesses and necrotic glioblastomas are listed in the Table. On SWI, hypointense rims were present in all $12 \mathrm{ab}-$ scesses, with 11 (91.7\%) of these being complete and 9 (75\%) being smooth. In 11 abscesses, the hypointense rims and the contrast-enhancing rims corresponded to the same locations (Fig $1 C,-D$ ). In 1 abscess, the hypointense rim and the contrast-enhancing rim partially overlapped. Part of the hypointense rim was inner to the contrast-enhancing rim. Of the glioblastomas, 15 of 20 (75\%) had SWI hypointense rims, of which 13 (86.7\%) were incomplete (Fig 2C) and 13 (86.7\%) were irregular (Fig 2C). The hypointense rims were inner to the contrast-enhancing rims in 9 necrotic glioblastomas (Fig $2 D$ ) and had the same locations as the contrast-enhancing rims in 2. The hypointense rims and the contrast-enhancing rims partially overlapped in 4 necrotic glioblastomas. Some parts of the hypointense rims were inner to the contrastenhancing rims. Figure 3 shows a necrotic glioblastoma without a T2-weighted or SW hypointense rim. A dual rim sign was present in 9 abscesses (Fig $1 C,-D$ ) but absent in all of the necrotic glioblastomas.

The difference in the prevalence of susceptibility-weighted hypointense rims between abscesses and glioblastomas was marginally significant $(P=.059)$. There were significant differences between abscesses and necrotic glioblastomas in the border types $(P<.001)$, contour $(P<.001)$, and locations $(P<.001)$ of the susceptibility-weighted hypointense rims as well as the prevalence of the dual rim sign $(P<.001)$. The sensitivity, specificity, accuracy, and positive and negative predictive values in differentiating abscesses from necrotic glioblastomas for each imaging feature that was significantly different are shown in the Table.

\section{Discussion}

Our study showed that hypointense rims on T2WI could not differentiate abscesses from necrotic glioblastomas. In contrast, abscesses and necrotic glioblastomas demonstrated significant differences in the border types, location, and contour of their hypointense rims on SWI. These differences on SWI suggest that the magnetic susceptibility in the lesion borders was probably from different sources and may represent different histologic components. To the best of our knowledge, this is the first report investigating the usefulness of SWI in differentiating abscesses from necrotic glioblastomas. 

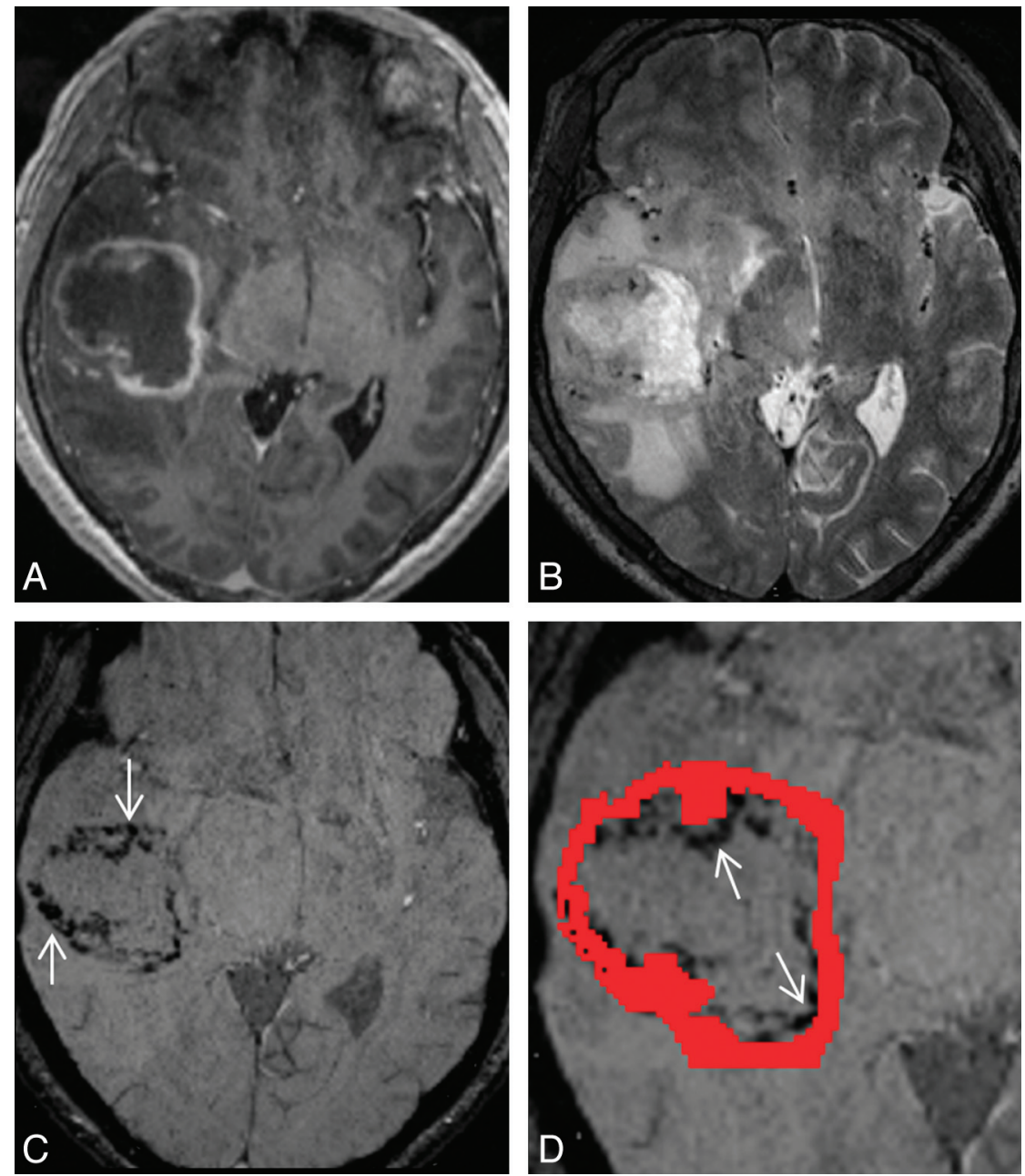

Fig 2. A 57-year-old man with a right temporal necrotic glioblastoma. $A$, Transverse contrast-enhanced MPRAGE shows a rim-enhancing mass in the right temporal lobe. $B$, On the transverse

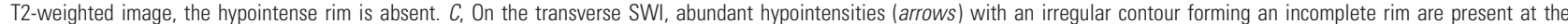
lesion margin. $D$, On the close-up view of SWI with a contrast-enhancing rim (red region of interest) overlaid, some of these hypointensities (arrows) are inner to the contrast-enhancing rim, while some are overlapping the contrast-enhancing rim.
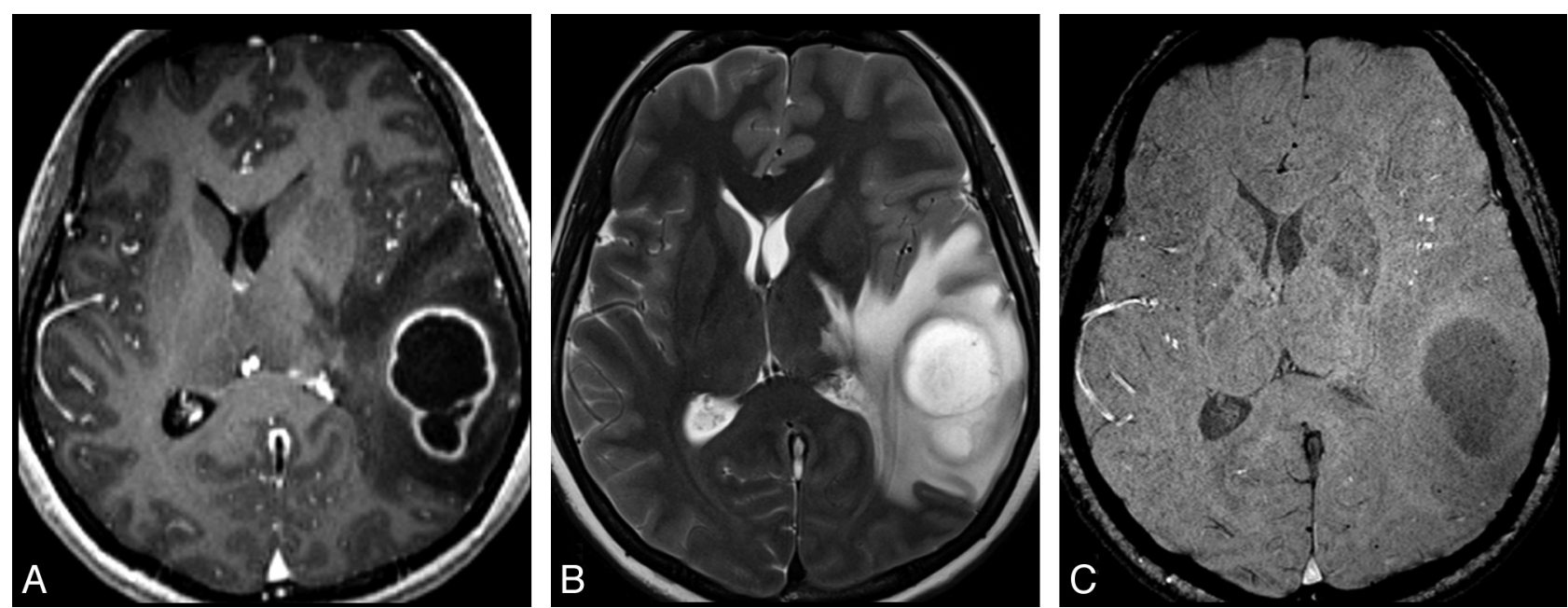

Fig 3. A 40-year-old woman with a left temporal necrotic glioblastoma. $A$, Transverse contrast-enhanced MPRAGE shows a rim-enhancing mass in the left temporal lobe. The hypointense rim is absent on both transverse $\mathrm{T} 2 \mathrm{~W}(B)$ and $\mathrm{SW}(C)$ images.

The high sensitivity to magnetic susceptibility and submillimeter resolution of SWI allowed the lesion borders of abscesses and necrotic glioblastomas to be evaluated in detail.
On SWI, the hypointense rims of abscesses tended to be complete and smooth and to have same locations as the contrastenhancing rims. It has been shown that the contrast-enhanc- 
ing rims of brain abscesses on CT and MR imaging correspond to the abscess capsule on histology. ${ }^{6,12}$ The magnetic susceptibility in the abscess capsule is thought to result from the production of paramagnetic free radicals by macrophages. ${ }^{6}$ In contrast, necrotic glioblastomas featured hypointense rims that were incomplete, irregular, and frequently found at the inner aspect of the contrast-enhancing rims. We speculate that the hypointense rims found in glioblastomas were likely due to random deposition of hemorrhagic products at the edge of the necrotic cavity.

The dual rim sign was the most specific imaging feature differentiating pyogenic brain abscesses from necrotic glioblastomas on SWI. To the best of our knowledge, this finding has not been reported. Although it was not mentioned by the authors, it can be retrospectively found in the pictorial essay published by Ong and Stuckey ${ }^{10}$ and in another study by Gupta et al. ${ }^{13}$ Because the dual rim sign can be found in different studies, we believe it is a reliable imaging finding that can be used to differentiate abscesses from necrotic glioblastomas.

Microscopically, there is a zone of granulation tissue between the necrotic center and fibrocollagenous capsule of brain abscesses. ${ }^{12}$ The granulation tissue, found at the very periphery of necrotic center, is composed of inflammatory cells, large foamy histiocytes, and fibroblasts. ${ }^{12}$ With image coregistration, the hyperintense rims were found at the most peripheral zone of abscess cavities and were deep to the contrast-enhancing rims. Therefore, we speculate that the hyperintense rims probably represent granulation tissue.

In the postprocessing of SWI data, the magnitude and phase information are combined. The signal-intensity changes on SWI arise from both T2 and susceptibility differences between tissues. ${ }^{8}$ The hyperintense rims could, therefore, be due to relatively higher T2 or lower magnetic susceptibility compared with the center cavities and fibrocollagenous capsules. Although the cause of hyperintense rims cannot be determined, their presence indicates that SWI may demonstrate a greater level of abscess organization than other MR imaging pulse sequences. Therefore, besides complementing conventional MR imaging and DWI in differentiating abscesses from necrotic glioblastomas, SWI may potentially serve as a clinical surrogate marker, monitoring the treatment response of brain abscesses. Further studies are needed to investigate the role of SWI in the management of patients with brain abscesses.

There were 2 steps in our imaging analysis that led to successful differentiation between abscesses and necrotic glioblastomas. In most of the previous studies, ${ }^{8,9}$ the SWI data were typically viewed by using a mIP over 4 images to enhance the visibility of voxels with low signal intensity (ie, high magnetic susceptibility). A caveat of this is that voxels with high signal intensity may become less obvious following mIP. In our study, we analyzed original SWI data without mIP so that both the hyperintense and hypointense rims of the dual rim sign could be detected on visual inspection. Furthermore, the original SWI data without MIP also allowed precise image coregistration and subsequent determination of the locations of the hypointense rims relative to the contrast-enhancing rims.

Because aspiration of pus plus antibiotics is the preferred treatment method for brain abscesses, histopathologic examination of the abscess margins may not be available unless en bloc resection is performed. Therefore, our study is limited by lack of direct histopathologic correlation with SWI findings. Thus, we cannot definitely state that the morphologic differences in lesion borders on SWI were due to histology components with different magnetic susceptibility. Despite this limitation, the lesion margins of abscesses and necrotic glioblastomas were different on SWI in our study.

\section{Conclusions}

SWI may be helpful in differentiating abscesses from necrotic glioblastomas based on the morphologic differences of their lesion margins. The dual rim sign is the most specific imaging feature distinguishing the 2 and is typically seen in pyogenic abscesses. SWI could potentially be helpful in the differentiation of brain abscesses from necrotic glioblastomas in the minority of times in which the evaluation with DWI of abscesses is made difficult by high ADC or when necrotic glioblastomas have central low ADC, which would be expected in pyogenic abscesses.

Disclosures: Mauricio Castillo_UNRELATED: Employment. American Society of Neuroradiology, Comments: American Journal of Neuroradiology, Editor-in-Chief, Royalties: Elsevier, Lippincott, Cambridge, Comments: book royalties.

\section{References}

1. Toh CH, Wei KC, Ng SH, et al. Differentiation of brain abscesses from necrotic glioblastomas and cystic metastatic brain tumors with diffusion tensor imaging. AJNR Am J Neuroradiol 2011;32:1646-51

2. Reddy JS, Mishra AM, Behari S, et al. The role of diffusion-weighted imaging in the differential diagnosis of intracranial cystic mass lesions: a report of 147 lesions. Surg Neurol 2006;66:246-50, discussion 50-51

3. Lee EJ, Ahn KJ, Ha YS, et al. Unusual findings in cerebral abscess: report of two cases. Br J Radiol 2006;79:e156-61

4. Hakyemez B, Erdogan C, Yildirim N, et al. Glioblastoma multiforme with atypical diffusion-weighted MR findings. Br J Radiol 2005;78:989-92

5. Reiche W, Schuchardt V, Hagen T, et al. Differential diagnosis of intracranial ring enhancing cystic mass lesions: role of diffusion-weighted imaging (DWI) and diffusion-tensor imaging (DTI). Clin Neurol Neurosurg 2010;112:218-25

6. Haimes A, Zimmerman R, Morgello S, et al. MR imaging of brain abscesses. AJR Am J Roentgenol 1989;152:1073-85

7. Schwartz KM, Erickson BJ, Lucchinetti C. Pattern of T2 hypointensity associated with ring-enhancing brain lesions can help to differentiate pathology. Neuroradiology 2006;48:143-49

8. Haacke EM, Mittal S, Wu Z, et al. Susceptibility-weighted imaging: technical aspects and clinical applications, part 1. AJNR Am J Neuroradiol 2009;30:1930. Epub 2009 Jan 8

9. Mittal S, Wu Z, Neelavalli J, et al. Susceptibility-weighted imaging: technical aspects and clinical applications, part 2. AJNR Am J Neuroradiol 2009;30: 232-52

10. Ong BC, Stuckey SL. Susceptibility weighted imaging: a pictorial review. J Med Imaging Radiat Oncol 2010;54:435-49

11. Robinson RJ, Bhuta S. Susceptibility-weighted imaging of the brain: current utility and potential applications. J Neuroimaging 2011;21:e189-204

12. Britt RH, Enzmann DR, Yeager AS. Neuropathological and computerized tomographic findings in experimental brain abscess. J Neurosurg 1981;55:590603

13. Gupta RK, Tomar V, Awasthi R, et al. T2*-weighted MR angiography substantially increases the detection of hemorrhage in the wall of brain abscess: implications in clinical interpretation. Neuroradiology 2012;54:565-72 